\title{
USING GENETIC ALGORITHMS TO OPTIMIZE THE DISPATCHING SCHEDULE OF RMC CARS
}

\author{
Chung-Wei Feng ${ }^{1}$, Hsien-Tang $\mathrm{Wu}^{2}$ \\ ${ }^{1}$ Assistant Professor, Dep. of Civil Eng., National Cheng Kung Univ., Tainan, Taiwan \\ e-mail: cfeng@mail.ncku.edu.tw \\ ${ }^{2}$ Graduate Student, Dep. of Const. Eng., Chaoyang Univ. of Technolog,Taichung, Taiwan \\ e-mail:s8811608@mail.cyut.edu.tw
}

\begin{abstract}
Effective and efficient dispatch of Ready Mixed Concrete (RMC) cars to construction sites is an important issue for both RMC plant and construction site managers because of the timeliness requirement of RMC. To develop an efficient utilization plan for dispatching RMC cars, RMC plant manager must consider both timeliness and flexibility while meeting the schedule at the job site. However, a system approach to such problem has seldom been done due to the facts of the complexity and uncertainty involved at the job site. For example, a RMC plant manager usually wants to keep dispatching RMC cars as many as possible to maximize production and profits of the plant. But delays of casting concrete at the job site and loading limits of RMC cars required by the law often cause that the schedule of dispatching RMC cars fails to meet the above goal and needs to be changed from time to time. Therefore, a system approach to such problem is necessary. This paper presents a new model, which is based on Genetic Algorithms (GAs) to optimize the dispatching schedule of RMC cars. The results show that GAs can easily transfer such problem into the Traveling Salesman Problem (TSP) and have satisfactory outcomes. In addition, a computer program written with Visual Basic ${ }^{\circledR}$ is developed to help the RMC plant engineers dispatch RMC cars.
\end{abstract}

KEYWORDS: Optimization, Genetic Algorithms, RMC Cars Dispatching

\section{INTRODUCTION}

Ready Mixed Concrete (RMC) was first introduced into the construction industry in the early twentieth century and has been ever since widely employed. Because of the rapid solidification of the concrete, RMC cars were developed for caring RMC to remote job sites. However, to dispatch RMC cars, the RMC plant manager must consider both timeliness and flexibility while meeting the schedule at the construction job site. However, a system approach to such problem has seldom been done due to the facts of the complexity and uncertainty involved at the job site. For example, a RMC plant manager usually wants to dispatch RMC cars as many as possible to maximize production and profits of the plant. But delays of casting at the job site and loading limits of RMC cars required by the law often cause that the schedule of dispatching RMC cars fails to meet the above goal and needs to be changed from time to time. Most research related to RMC focus on improving and assuring the quality of RMC. Examples are [3][5]. Not much research has been done for dispatching RMC cars. Considering the fact that saving time and reducing cost for operating the $\mathrm{RMC}$ plant, a system approach to such problems is necessary.

1.1 Ready mixed concrete industry

Several characteristics, different from other industries, of the operation in the RMC industry need to be addressed before developing the model.

A. No finished products are in stock

Because the quick solidification of the concrete, RMC cannot be manufactured in advance and should to be produced at the time the job site manager requires the dispatching. There are no finished products in stock and the dispatching schedule of RMC cars could be affected by the status at the job site and the RMC plant. Therefore, it is hard for the RMC plant manger to decide the dispatching schedule of the RMC cars to the different job sit in advance.

B. Limited service areas of RMC cars

To keep the quality and is required by the law, RMC has to be cast within the 1.5 hours after being 
produced. Therefore, the service areas of RMC cars are limited and should be considered as a main factor for dispatching RMC cars.

\section{GENETIC ALGORITHMS}

Genetic Algorithms (GAs) are search algorithms developed by Holland in 1975, which are based on the mechanisms of natural selection and genetics to search through decision space for optimal solutions [2]. The metaphor underlying GAs is natural selection. In evolution, the problem each species faces is to search for beneficial adaptations to the complicated and changing environment. In other words, each species has to change its chromosome combination to survive in the real world. In GAs, a string represents a set of decisions (chromosome combination), a potential solution to a problem. Each string is evaluated on its performance with respect to the fitness function (objective function). The ones with better performance (fitness value) are more likely to survive than the ones with worse performance. Then the genetic information is exchanged between strings by crossover and mutation. The result is a new generation with (usually) better survival abilities. This process is repeated until the strings in the new generation are identical, or certain termination conditions are met. Figure 1 (Adopted from [1]) shows the process of Genetic Algorithms

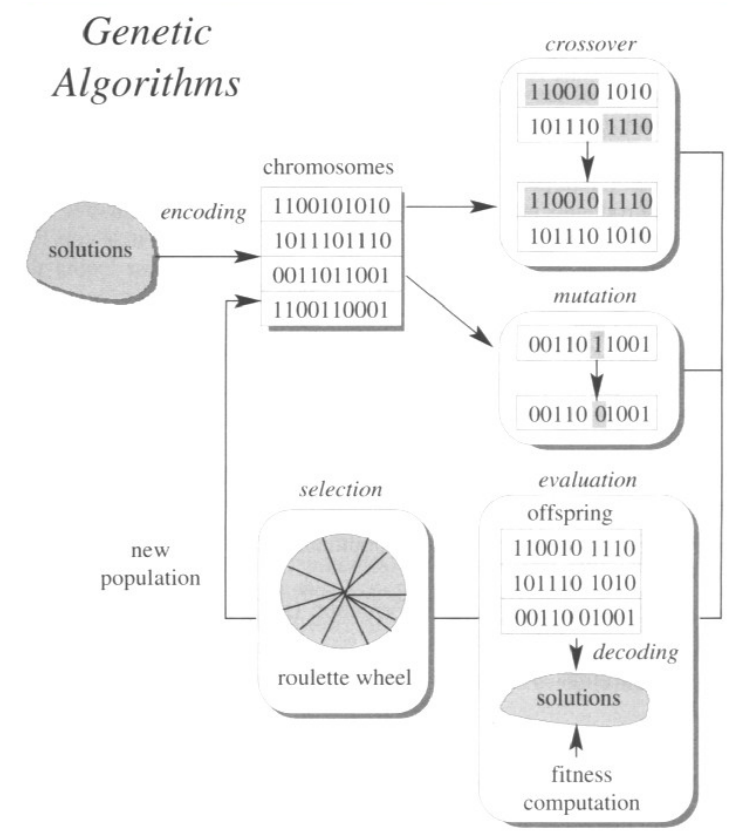

Figure 1. Process of Genetic Algorithms

In the past two decades, GAs have been successfully formulated to solve many science and engineering problems and have proved to be an efficient means of searching for the optimal solutions of large-scale optimization problems.

\section{GA FOR DISPATCHING RMC CARS}

There are any factors that would impact the dispatching schedule of RMC cars. To develop a model for dispatching schedule, we only focus on the factors that are important to dispatching RMC cars. In addition, these factors are assumed to be determined in advance.

\subsection{Factors impact the dispatching of RMC cars}

A. Traveling Time from the RMC plant to the job site

RMC cars usually travel from the RMC plant and the job site back and forth. The traveling time between the RMC plant and the job site affects the dispatching schedule of the RMC cars. Usually, RMC cars are assigned to the same job site for one day. However, it is possible that the RMC plant manger can use the floats between assigning the RMC car to the same job site to assign the RMC car to the different job site, which maximizes the productivity of the RMC plant. Therefore, traveling time from the $\mathrm{RMC}$ plant to the job site is an important factor of deciding the dispatching schedule of the RMC cars. In addition, RMC has to be delivered to the job sit and cast within 1.5 hours; therefore, the traveling time from the RMC plant to the job site should be considered within the dispatching model.

B. Working time at the job site

The working time of the RMC car at the job site varies with the types of the construction activities. As a result, the dispatching schedule of RMC cars depends on the working time of the activities at construction site. For example, the faster unloading of concrete at the job site is, the shorter interval time between the RMC cars to the construction job site is required. If the RMC cars cannot satisfy the needs at the job site, it is possible that the whole job site is idle and waits for the arrival of the RMC cars. Tremendous amount of time is wasted, which does not only impact the productivity at the job site but also the RMC plant. Therefore, how to satisfy the needs at the construction job sites and maximize the productivity of the RMC plant is an important issue to the RMC plant manager.

C. Amount of concrete needed at the job site

The amount of concrete needed at the job site usually is defined as "the one-day amount of concrete needed at the job site." The more amount of concrete needed at the job site, the more number of RMC cars is required to the job site. The RMC manager may assign as many RMC cars to the busiest job site as possible. However, such approach may cause many $\mathrm{RMC}$ cars waiting at the job site to unload the concrete. Thus, dispatching RMC cars has to 
consider the amount of concrete needed at the job site.

D. Single loading limit of a RMC car

The single loading limit of the RMC car required by the law depends on the condition of the path to the job site. If the condition of road is bad or the required law of the local government is more restricting the loading limit of a RMC car is reduced. Consequently, the number of RMC cars to the job site increases.

\section{E. Mixing time of concrete}

The mixing time of concrete affects the time that RMC can depart to the job site. As the rule of thumb, the more concrete required, the more time needed to mix, which also impact the dispatching of the RMC car.

\subsection{The genetic algorithms Model}

\section{A. Chromosome structure:}

Although emergent requests of concrete from the job sites could happen if the required amounts of concrete at the job site is under-estimated, the job site manager usually order the amount of the concrete needed before the day of casting concrete. Therefore, it is reasonable to assume that the RMC plant manager knows the required amounts of the concrete for different job sites in advance; that is, the numbers of the RMC cars needed for the different job sites are pre-determined. Determining the dispatching schedule of the RMC cars is the same as to decide the permutation of assigning RMC cars to different job sites. This decision making process is very similar to solving the traveling salesman problem (TSP). For TSP, the objective is to find the schedule of the salesman, which minimizes the traveling costs of the salesman, while ensure that salesman visits each required city one time only. As described in [4], the search space for the TSP is a set of permutations of $n$ cities. Any single permutation of $\mathrm{n}$ cities yields a solution. The size of the search space is $n$ !. Therefore, the chromosome structure of a string in this genetic algorithm can be defined as the following:

The length of a string is the total number of times of the RMC cars dispatched from the RMC plant. For example, if the there are 4 job sites and each needs 2 RMC cars to deliver concrete, the total length of the string is 8 . In addition, the genes of a string represent their permutations. There are two strings (S1 and S2) in Figure 2, in which (ij) represents the jth car assigned to job site i. Random keys representation is used to decide the permutations of the RMC cars. For example, in string 1 , the permutation is (42), (21), (31), (12), (41), (22), (32) and (11). That is, the RMC cars are orderly assigned to job site 4, 2, 3, 1, 4, 2, 3, and 1 .

\begin{tabular}{|c|c|c|c|c|c|c|c|c|}
\hline$(\mathrm{i} \mathrm{j})$ & 11 & 12 & 21 & 22 & 31 & 32 & 41 & 42 \\
\hline S1 & 0.81 & 0.22 & 0.08 & 0.62 & 0.20 & 0.80 & 0.35 & 0.04 \\
\hline S2 & 0.95 & 0.24 & 0.30 & 0.23 & 0.34 & 0.75 & 0.98 & 0.81 \\
\hline
\end{tabular}

Figure 2. Example of the chromosome structure B. The fitness function

As described in section 3.1, the manager of RMC plant usually wants to maximize the productivity of the RMC plant, which indicates that the RMC cars should be as busy as possible. Both waiting time of the RMC cars and idle time at job site is treated as costs and should be avoided. Therefore, the fitness function of the proposed genetic algorithm is defined as minimizing the total waiting time of RMC cars and idle time at the job site. The fitness value of the string can be decided by the following formulas.

Minimize $\Sigma\left(\mathrm{CWT}_{\mathrm{ij}}-\mathrm{SWT}_{\mathrm{ij}}\right)$

in which,

If $\mathrm{SWT}_{\mathrm{ij}}=0$ then $\mathrm{CWT}_{\mathrm{ij}}=0$. or

If $\mathrm{SWT}_{\mathrm{ij}}<0$ then $\mathrm{CWT}_{\mathrm{ij}}=0$. or

If $\mathrm{CWT}_{\mathrm{ij}}>0$ then $\mathrm{SWT}_{\mathrm{ij}}=0$.

$\mathrm{SWT}_{\mathrm{ij}}$ and $\mathrm{CWT}_{\mathrm{ij}}$ can be obtained from

$T_{i j}=W T_{i j-1}-\left(G T_{i}+M T_{i j}-M T_{i j-1}\right)$

If $\mathrm{T}_{\mathrm{ij}}<0$ then

$\mathrm{SWT}_{\mathrm{ij}}=\mathrm{T}_{\mathrm{ij}}, \mathrm{CWT}_{\mathrm{ij}}=0, \mathrm{WT}_{\mathrm{ij}}=\mathrm{DT}_{\mathrm{i}}$

If $\mathrm{T}_{\mathrm{ij}}>=0$ then

$\mathrm{CWT}_{\mathrm{ij}}=\mathrm{T}_{\mathrm{ij}}, \mathrm{SWT}_{\mathrm{ij}}=0, \mathrm{WT}_{\mathrm{ij}}=\mathrm{DT}_{\mathrm{i}}+\mathrm{T}_{\mathrm{ij}}$

where

$\mathrm{SWT}_{\mathrm{ij}}$ : the idle time at job site $\mathrm{i}$, waiting for the jth RMC car.

$\mathrm{CWT}_{\mathrm{ij}}$ : the waiting time of the jth RMC car at job site i for unloading.

WTij-1:the waiting time of the j-1th RMC car for job site i.

$\mathrm{GT}_{\mathrm{i}}$ :the traveling time from the RMC plant to the job site i.

$\mathrm{DT}_{\mathrm{i}}$ : the unloading time of RMC car at job site $\mathrm{i}$.

$\mathrm{MT}_{\mathrm{ij}}$ : the departure time of the jth RMC car to the job site i.

\section{TEST EXAMPLE AND RESULT}

An example is used to describe the proposed genetic algorithm for dispatching RMC cars. The condition information of the job sites is listed in Table 1. Table 2 shows the mixing time for different amounts of RMC. 
$2|0.95| 0.24|0.30| 0.23|0.34| 0.75|0.98| 0.81|0.97| 0.02$

Figure 3. Random keys of two strings

\begin{tabular}{c|cccccc} 
Job site & 1 & 2 & 3 & 4 & 5 & 6 \\
\hline GT & 21 & 43 & 36 & 20 & 27 & 60 \\
DT & 10 & 5 & 7 & 15 & 23 & 12 \\
WT & 0 & 0 & 0 & 0 & 0 & 0 \\
DQ & 16 & 100 & 87 & 230 & 30 & 350 \\
SQ & 0 & 88 & 75 & 200 & 22 & 333 \\
LQ & 6 & 9 & 9 & 6 & 9 & 9 \\
ST & 1 & 1 & 1 & 0 & 1 & 1
\end{tabular}

Table1. The information of the job sites

DQ: Required amount of concrete at the job site.

SQ: Amount of concrete has been delivered to job site.

LQ: Loading limit of the concrete

ST: Status of the job site; 1 means job site is in activity, 0 means job site is idle.

(Note: The amount of the concrete are in $\mathrm{M}^{3}$, the time scale is in minutes)

\begin{tabular}{c|ccccccccc}
$\mathrm{Q}$ & 1 & 2 & 3 & 4 & 5 & 6 & 7 & 8 & 9 \\
\hline $\mathrm{MT}$ & 2 & 2 & 2 & 3 & 3 & 3 & 4 & 4 & 4
\end{tabular}

Table 2. Mixing time for the different amount of concrete

\subsection{Chromosome structure and fitness value}

From Table 1, the remaining number of RMC cars for the job sites can be determined and is listed in Table 3.

\begin{tabular}{c|cccccc} 
Job site & 1 & 2 & 3 & 4 & 5 & 6 \\
\hline \# of Cars & 3 & 2 & 2 & 0 & 1 & 2
\end{tabular}

Table 3. Remaining number of RMC cars for each job site.

The remaining number of $\mathrm{RMC}$ cars for the job sites is determined by (DQ-SQ)/LQ. If (DQ-SQ)/LQ is not an integer then an additional car is required. No RMC cars are required for the job site 4 because job site 4 is idle. From Table 3, the length of the chromosome can be obtained by summing up the remaining number of RMC cars, which is 10. As described in section 3.2, the initial parent population is generated by using random keys representation. Figure 3 shows two strings with random keys.

\begin{tabular}{|c|c|c|c|c|c|c|c|c|c|c|}
\hline (ij) & 11 & 12 & 13 & 21 & 22 & 31 & 32 & 51 & 61 & 62 \\
\hline 1 & 0.81 & 0.22 & 0.08 & 0.62 & 0.20 & 0.80 & 0.35 & 0.04 & 0.82 & 0.96 \\
\hline
\end{tabular}

After arrange (ij) in ascending order based on their random keys, the dispatching schedules of the RMC cars to the job sites of two strings are determined and shown in Figure 4.

\begin{tabular}{|c|c|c|c|c|c|c|c|c|c|}
\hline 1 & 2 & 3 & 4 & 5 & 6 & 7 & 8 & 9 & 10 \\
\hline 51 & 13 & 22 & 12 & 32 & 21 & 31 & 11 & 61 & 62 \\
\hline 62 & 21 & 12 & 13 & 22 & 31 & 51 & 11 & 61 & 32 \\
\hline
\end{tabular}

Figure 4. Dispatching schedules of two strings.

Based on the required mixing time of the amount of concrete in Table 2 and the remaining amount of concrete needed for the different job sites, the mixing time of the concrete is summarized in Table 4.

\begin{tabular}{c|cccccccccc} 
Site & 5 & 1 & 2 & 1 & 3 & 2 & 3 & 1 & 6 & 6 \\
\hline $\mathrm{Q}$ & 8 & 6 & 9 & 6 & 9 & 3 & 3 & 4 & 9 & 8 \\
$\mathrm{MT}$ & 4 & 3 & 4 & 3 & 4 & 2 & 2 & 3 & 4 & 4
\end{tabular}

Table 4. Remaining amounts of concrete needed for job sites and their mixing time at the RMC plant.

The waiting time for each RMC car of string 1 can be determined by Table 3 and (2), and is listed in Table 5. In addition, the fitness value of the string 1 is 89 .

\begin{tabular}{c|cccccccccc}
$\mathrm{S}$ & 5 & 1 & 2 & 1 & 3 & 2 & 3 & 1 & 6 & 6 \\
\hline $\mathrm{WT}$ & -4 & -7 & -11 & 3 & -18 & -4 & 3 & 2 & -29 & 8
\end{tabular}

Table 5. Waiting time of each RMC car in string 1. (Note: negative means that the job site waits for the $\mathrm{RMC}$ car)

\subsection{Crossover and mutation}

Crossover and mutation could destroy the strings and make them illegal. It is because that a string which has two genes with the same number is not allowed for permutation representation. Therefore, repairing processes are required to maintain the strings in permutation presentation. Partial-Mapped Crossover (PMX) is used in this research. PMX was proposed by Goldberg and Lingle (adopted from [1]). It can be viewed as an extension of two-point crossover for the binary string to permutation representation. Example of two legal strings is shown in Figure 5. The genes in the shaded section are exchanged for crossover. Figure 6 shows the illegal offspring after crossover. 


\begin{tabular}{|l|l|l|l|l|l|l|l|l|l|}
\hline 51 & 13 & 22 & 12 & 32 & 21 & 31 & 11 & 61 & 62 \\
\hline 62 & 21 & 12 & 13 & 22 & 31 & 51 & 11 & 61 & 32 \\
\hline
\end{tabular}

Figure 5. Example of two parent strings

\begin{tabular}{|l|l|l|l|l|l|l|l|l|l|}
\hline 51 & 13 & 22 & 13 & 22 & 31 & 31 & 11 & 61 & 62 \\
\hline 62 & 21 & 12 & 12 & 32 & 21 & 51 & 11 & 61 & 32 \\
\hline
\end{tabular}

Figure 6. Example of two illegal offspring

The repairing process is used to legalize the two illegal offspring. First, find the mapping relations between two selected (shaded) areas. That is, $13 \leftrightarrow 12,22 \leftrightarrow 32$, and $31 \leftrightarrow 21$. Then change the genes which are not shaded but with the same number in the shaded area to the mapping number. The legalized offspring is shown in Figure 7.

\begin{tabular}{|l|l|l|l|l|l|l|l|l|l|}
\hline 51 & 12 & 32 & 13 & 22 & 31 & 21 & 11 & 61 & 62 \\
\hline 62 & 31 & 13 & 12 & 32 & 21 & 51 & 11 & 61 & 22 \\
\hline
\end{tabular}

Figure 7. Legal string after PMX

Insertion mutation is used in this research. First, randomly select a gene and insert the gene to a randomly selected position. An example is shown in Figure 8 .

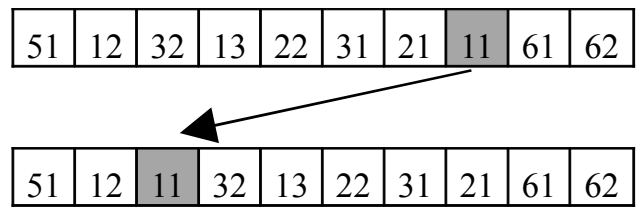

Figure 8. Example of mutation

\subsection{Result}

The result from the tested example, shown in Figure 9, shows that the proposed genetic-based model can quickly generate the dispatching schedule of the RMC cars. The optimal schedule is 3531621261. In addition, since the problem is similar to TSP, the potential solution space for the test example can be determined, which is $10 !=3628800$. With the population size of 100 and 200 generations, the proposed genetic algorithm search through only $0.55 \%$ solution space. Such results reveal that using the proposed genetic algorithm in this research is a promising approach to solve the dispatching schedule of the RMC cars.

\subsection{Computer implementation}

A prototype program named RMCGA is developed in this study to test the proposed model. RMCGA is coded with Visual Basic ${ }^{\circledR}$ and shown in
Figure 10. Users are only asked to enter the required amounts of concrete for the job sites and the RMCGA can then generate the formula and find the optimal schedule on its own.

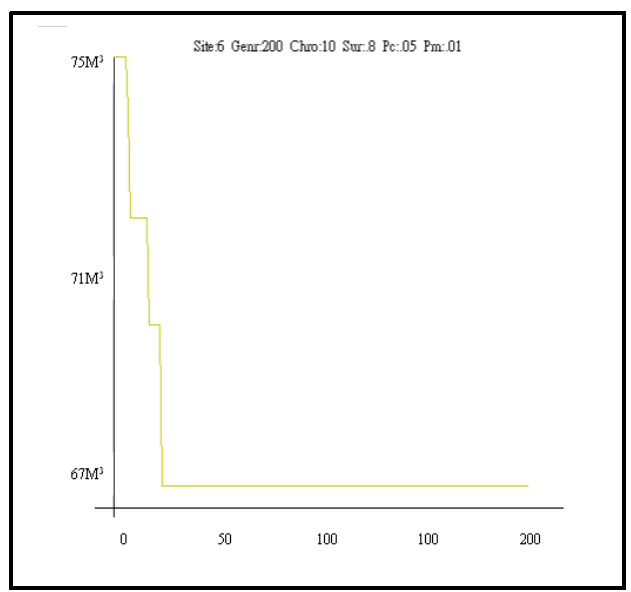

Figure 9. Result of the tested example

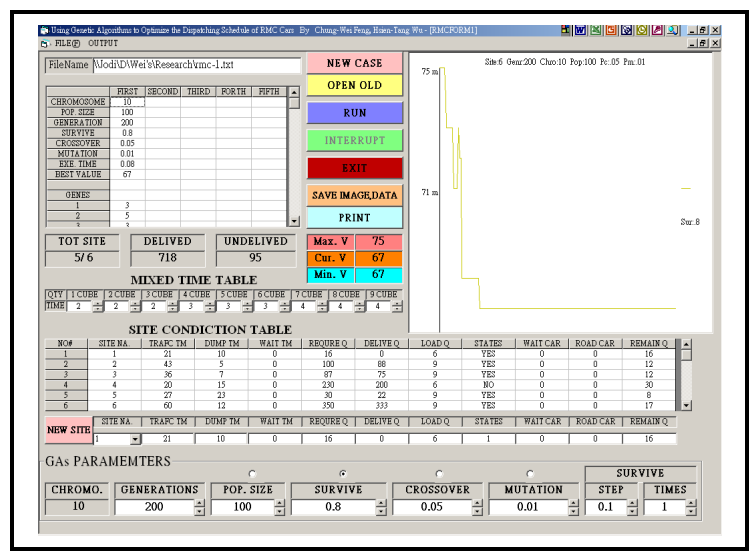

Figure 10. Interface of RMCGA

\section{CONCLUSIONS}

Several facts have been concluded in this research. First, a system approach to dispatch RMC cars is taken in this research. Based on the proposed model, the RMC plant manager can schedule their RMC cars more effectively. Second, a genetic algorithm for solving dispatching schedule of the RMC cars shows promising results. The genetic algorithm proposed in this research can generate the optimal schedule in small number of iterations. Therefore, the RMC plant manager can quickly decide the schedule for the RMC cars if the unexpected loading required for the job site is happened. Third, an user-friendly computer program is developed, which makes the proposed model be more applicable to the RMC industry. However, several issued are still up in the air and need to be solved in the future study.

A. The parameters used in this study are assumed 
to be certain and known in advance. Fussy theory or probability-based approach are expected to resolve this matter.

B. The efficiency of the crossover and mutation used in the proposed genetic algorithm can be improved. Similar to the development in using Genetic Algorithms to solve TSP, the genetic operators used in this research are expected to be improved. Other genetic operator, such as $\mathrm{CX}, \mathrm{OX}$, and heuristic crossover will be tested. Development of the new genetic operator will also be conducted.

Ready Mixed Concrete (RMC) has been employed in the construction industry since the early twentieth century. With the study of manage ring $\mathrm{RMC}$ cars the RMC plant can be more productive and so is the construction industry.

\section{REFERENCES}

[1] Gen, M., Cheng. R, Genetic Algorithms \& Engineering Design, John Wiley \& Sons, 1997.

[2] Goldberg, D.E., Genetic algorithms in search, optimization and machine learning, Addition-Wesley, Reading, MA., 1989.

[3] Khalaf Rejeb, Saeed, "Improving compressive strength of concrete by a two-step mixing method", Cement and Concrete Research, Vol. 26, No.4, pp. 585-592, 1996.

[4] Michalewicz, Z., Genetic Algorithms + Data Structures = Evolution Program, 3th edition, Springer, 1996.

[5] Zain, M.F.M., Safiuddin, M., Yusof, K.M, “A study on the properties of freshly mixed high performance concrete", Cement and Concrete Research, Vol. 29, No. 9, pp. 1427-1432, 1999. 